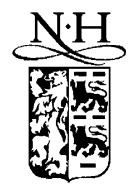

ELSEVIER

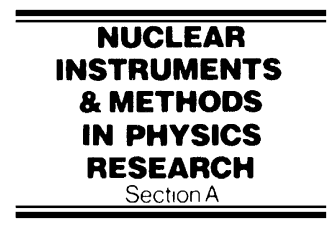

www.elsevier.nl/locate/nima

\title{
Method for effective dead time measurement in counting systems
}

\author{
F.L.R. Vinagre*, C.A.N. Conde \\ FCTUC, Departamento de Fisica, Universidade de Coimbra, P-3004-516 Coimbra, Portugal
}

Received 30 June 2000; received in revised form 13 September 2000; accepted 7 October 2000

\begin{abstract}
The counting losses introduced by the dead time of a counting system are a limiting factor in counting measurements. The purpose of this work is to report an efficient method for the measurement of the effective dead time of a counting system and to characterize its dead time behavior, providing a way to investigate each experimental situation. The method, which we designate as Delayed and Mixed Pulses method, is based on the artificial piling-up of detector pulses with electronic pulses delayed by a specific time interval. It is applicable to the measurement of the effective dead time of a counting system, including both pile-up effects and the dead time characteristics of the elements of the counting chain. With counting systems relying on gaseous radiation detectors, we achieved a standard uncertainty of about $5-10 \%$ in the dead times measured. (C) 2001 Published by Elsevier Science B.V.
\end{abstract}

PACS: $07.85 .-\mathrm{m} ; 29.40 .-\mathrm{n} ; 07.50 .-\mathrm{e}$

Keywords: Pulse pile-up; Dead time behavior; Resolution time; Counting losses; Radiation detectors; Absolute counting measurements.

\section{Introduction}

The resolution time or dead time of a counting system affects inevitably the counting of events from a random process. When two or more events occur in a time interval shorter than the resolution time, they are seen as a single event and the measured count-rate is lower than the true countrate. The consequent counting inefficiencies assume vital importance in absolute counting measurements [1-3]. Moreover, the pulse shape is distorted

\footnotetext{
*Corresponding author.

E-mail address: fleonor@saturno.fis.uc.pt (F.L.R. Vinagre).
}

due to this effect, which affects applications such as radiation spectroscopy at high count-rates and timing measurements [4].

A standard approach to correct the counting inefficiencies is to characterize the dead time behavior of the counting system as a particular function of the system dead time [4]. Two standard types of dead time behaviors are usually considered: the extending (or paralyzable) and the nonextending (or non-paralyzable) ones. In another approach, the correction is obtained by inserting an electronic unit with a fixed dead time in the analogue or digital part of the signal chain, with a dead time longer than the dead time of any other unit of the chain [5-7]. One other technique is 
known as the pulser method and uses pulses from an electronic pulse generator, with known frequency, to mix them with detector pulses $[1,2$, 8-10]. Additional procedures to deal with dead time problems involve the detection of pile-up pulses with electronic PUR circuits (Pile-Up Rejectors) [1,11,12], or their correction using digital-processing techniques [13].

Some of these methods can only be applied if the type and experimental value of the dead time of the counting system are known. The measurement of the dead time is traditionally done by methods based on the non linear dependence of the measured count-rate on the real count-rate, namely the decaying source or the two-source methods and their modifications $[4,6]$. The purpose of this work is to report a low time consuming method for the measurement of the effective dead time of a counting system and to characterize its dead time behavior. Since there is no reason why any real system should have any standard behavior, nor involve a single dead time, no general solution seems to exist to the dead time problem $[5,7,14]$. A reasonable approach is to investigate the correction to be applied for any experimental setup. A simple method is thus preferred.

\section{Experimental method}

We propose a method for the experimental determination of the effective resolution time of a counting system, which we designate as Delayed and Mixed Pulses (DMP) method. No spectra distortion is investigated in this work. We studied two measurement schemes in which the detector signal is passed through a pre-amplifier (PA) and a linear amplifier (A). Then the pulses are discriminated from noise and transformed into standard logic pulses with a pulse discriminator (PD) and counted with an electronic counter (EC) or else processed with a multi-channel analyzer (MCA).

The experimental setup of the DMP method is presented in Fig. 1. A pulse from the detector, corresponding to a random event, triggers an electronic pulse with a delay selected manually by the experimentalist in the electronic pulse generator. The selected delay is measured directly in

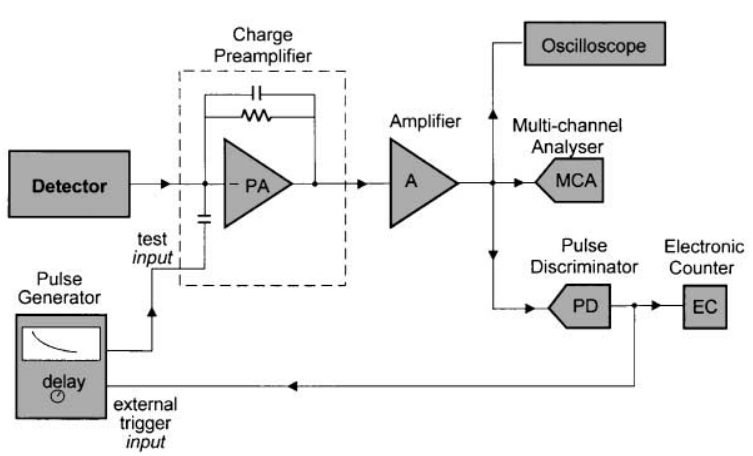

Fig. 1. Experimental setup for the measurement of the counting system effective dead time.

the oscilloscope display. This pulse is then fed to the test input of the PA to be shaped and processed exactly as the detector pulses and to be mixed with them.

The DMP method consists of recording the measured pulse count-rate $m$ at the output as a function of the delay between the detector pulses and the electronically generated ones. For a null delay the pulses are summed, giving rise to a pulse with the sum amplitude, and the counting system is unable to resolve the detector pulses from the electronic ones. By increasing the delay beyond the system effective dead time, the measured countrate increases quickly by a factor of 2 . For delays longer than this, the counting system is capable of resolving these events and the measured count-rate corresponds to the count-rate of all the pulses from the detector and all the electronically generated pulses, i.e. twice the detector count-rate is measured. Fig. 2 shows a digitized form of the pulses of a typical sequence. The effective dead time is obtained from the delay in the transition zone, at the point where the count-rate is the mean of the maximum and minimum total count-rate (Fig. 3).

The DMP method is basically different from those methods relying on mixing electronic and detector pulses, the source-generator method (SGM) [6] and the pulser method [1,2,8-10]. The DMP method is based on a time correlation between the pulses from the detector and those from the pulse generator, providing a way to measure directly the effective dead time of the 

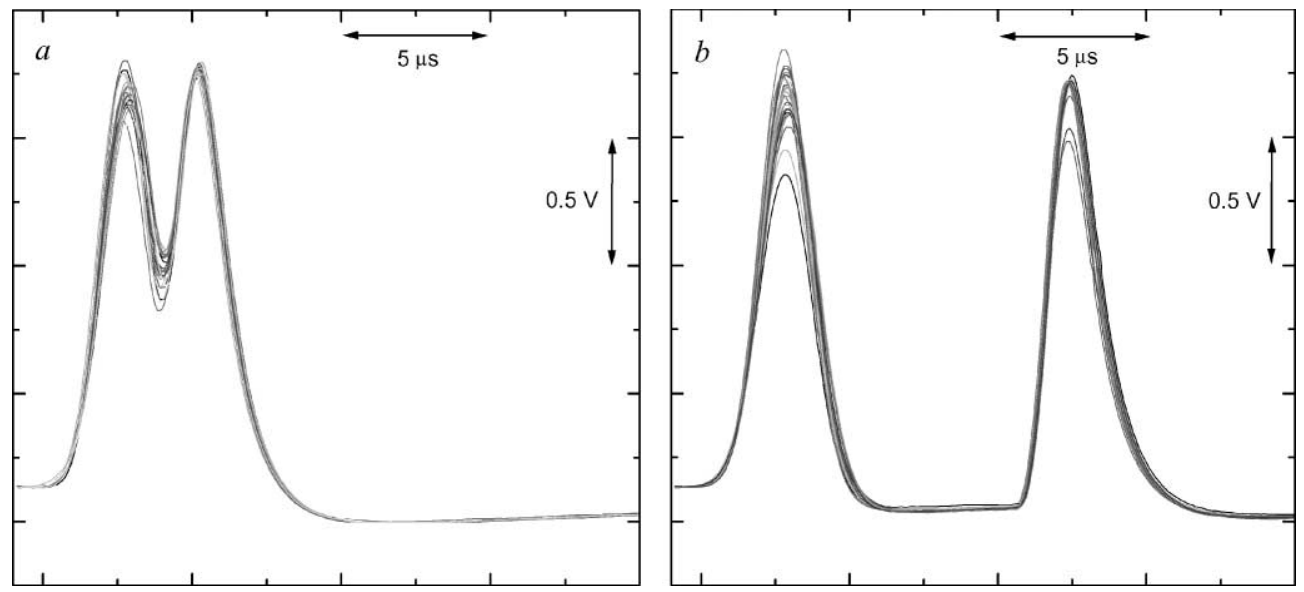

Fig. 2. Digitized form of the pulses (sampling rate of $1.25 \mathrm{MHz}$ ) of a typical sequence. The first set of pulses in each figure corresponds to $5.9 \mathrm{keV} \mathrm{X}$-ray events in a gas proportional scintillation counter filled with $\mathrm{Xe}-10 \%$ Ar. The pulses in the second set are generated electronically by the first pulses after a delay of $4 \mu$ s (a) or $10.5 \mu$ s (b).

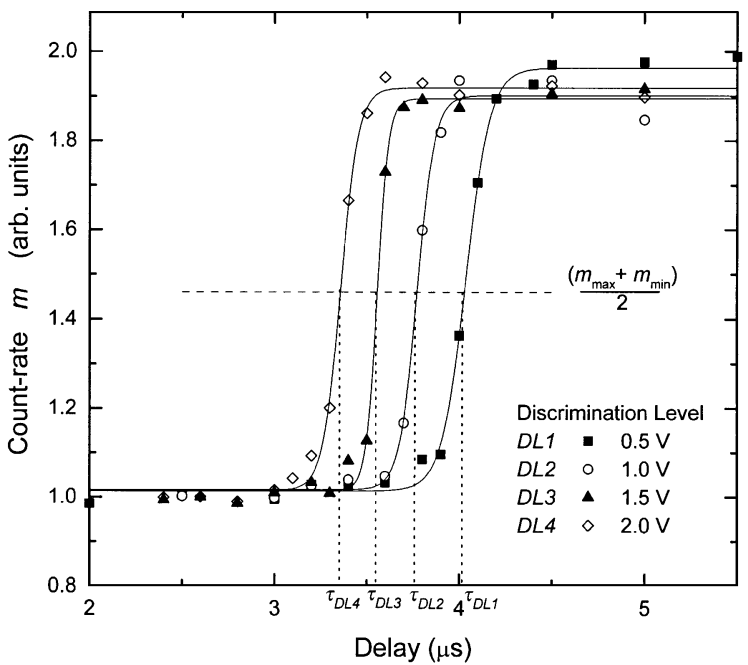

Fig. 3. Dead times for several discriminator levels (DL) of the pulse discriminator, with the GPSC $\rightarrow$ PD-EC counting system, with a pulse amplitude of $3.5 \mathrm{~V}$.

counting system. The DMP method can be applied to most counting systems using a radiation detector. It cannot be applied to the cases when the detector itself has an intrinsic dead time. In most radiation detectors, with an exception for the G-M tube, any event leads to one output detector pulse, even if it occurs after an infinitely small time interval after the previous event and thus no dead time is associated intrinsically to the detector. So, in this case, the losses in the counting measurements are due to the response of the counting system to the specific detector pulses. Our method, DMP method, allows this effective dead time measurement, which involves the dead time of the counting system and the pile-up between the detector pulses.

\section{Results and discussion}

To test the proposed method, we used a hybrid detector [15] with two operation modes: proportional counter (PC) and gas proportional scintillation counter (GPSC), filled with Xe or $90 \%$ $\mathrm{Ar}-10 \% \mathrm{Xe}$, detecting $5.9 \mathrm{keV} \mathrm{X}$-rays from a ${ }^{55} \mathrm{Fe}$ radioactive source. The GPSC and PC pulses are fed to Canberra PA, models 2005 and 2006, respectively. We used a Tennelec TC 243 gaussian A, a single channel analyzer Hewlett Packard, model HP5583A, in the PD mode, an EC constructed in our Physics Department and pulse generators BNC model PB-4 and Datapulse model 101. The MCA is a Nucleus PCA II. Except when otherwise specified, we used output amplifier pulses of unipolar shape and shaping-times of 
$0.5 \mu \mathrm{s}$, a PD discrimination level (DL) of $1.5 \mathrm{~V}$ and a count rate of about $10^{3} \mathrm{~s}^{-1}$.

We will here distinguish two cases: in the first one the pulses are counted with the PD-EC system and in the second one with the MCA.

Fig. 3 shows the measured dead times $\tau$ for several DLs of the PD. As expected, by increasing the delay beyond a certain extent, the PD-EC system becomes able to resolve the detector events from the electronic pulses and the measured countrate $m$ practically doubles. These results show a clear dependence of the dead time $\tau$ of the PD-EC counting system on the DL. Whenever the pulse amplitude increases above the DL, the PD triggers the EC, which adds one count to the measured number of counts. The PD can only resolve a second pulse if the first pulse has fallen below the DL. As we would expect, increasing $\tau$ values are obtained with decreasing DLs (Fig. 3), since the pulse time interval above the DL is shorter when the DL is higher.

The influence of the dead time $\tau$ of the PD-EC system on the detector count-rate was also investigated in the range $200-24100 \mathrm{~s}^{-1}$ (Fig. 4). No significant differences in the measured $\tau$ were detected within the uncertainty of our measurements. However, the transition is less abrupt for

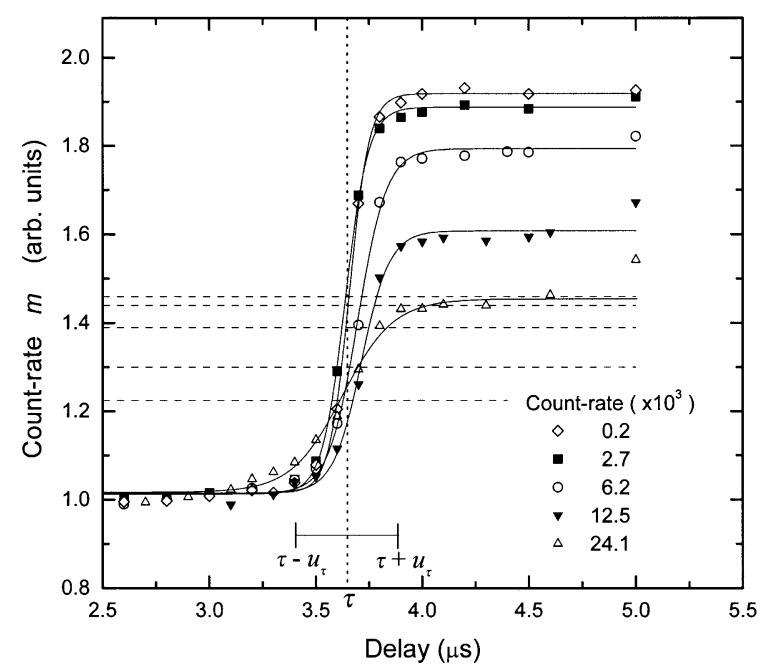

Fig. 4. Dead times for several detector counting-rates, with the GPSC (Xe-10\% Ar) $\rightarrow$ PD-EC counting system, with a pulse amplitude of $3.3 \mathrm{~V}$. high count-rates, which increases the variance in the measured dead time. Additionally, the measured count-rate $m$ rises to a value lower than twice the detector count-rate, decreasing as the count-rate increases. This effect, evidenced in Fig. 4, was investigated and was found to be mostly a consequence of the need of electronic pulses (generated by the BNC model PB-4 unit) with long decay times to prevent the system to fall into oscillation. The system takes some time to be ready to deliver another electronically generated pulse similar to the former one and so a detector pulse that arrives before that does not generate an electronic pulse, leading to single and not double pulses. This is the reason why at long delays the count-rate measured is lower than twice the detector count-rate and decreases as we increase the detector count-rate. Although no dependence of the $\tau$ on the count-rate was detected, we do not know the role it plays in more severe situations.

The previous analyses were also performed using a PC instead of the GPSC. The dead times in the $\mathrm{PC} \rightarrow \mathrm{PD}-\mathrm{EC}$ system are about $13 \%$ longer. The influence of the DL and the count-rate in the PC $\rightarrow$ PD-EC system lead to similar conclusions.

When the detector pulses are to be recorded and shaped by a MCA, besides the peak pile-up, which affects the counting efficiency, tail pile-up also occurs leading to distortion in the spectra due to deficient recorded pulse-heights. Also, the dead time of a MCA with a Wilkinson-type ADC is well known to be dependent on the acquiring channel. We applied the present method to measure the dead time of the GPSC $\rightarrow$ MCA counting system, for pulses of approximately constant pulse-height. Fig. 5 shows a sequence of spectra recorded by the MCA corresponding to a variable delay between the detector and their consequent electronic pulses, which have fixed amplitude. Also shown in Fig. 5, is the count-rate (area below the spectra) recorded in the MCA as a function of the delay.

The described experiences allowed the presentation of the method for the effective dead time measurement (DMP method). The uncertainty of the dead time measurement is caused by the uncertainty of the transition, $u_{\mathrm{t}}$, and by the uncertainty of the time measurement, $u_{\mathrm{m}}$, in the oscilloscope display. The first contribution $u_{\mathrm{t}}$ is 


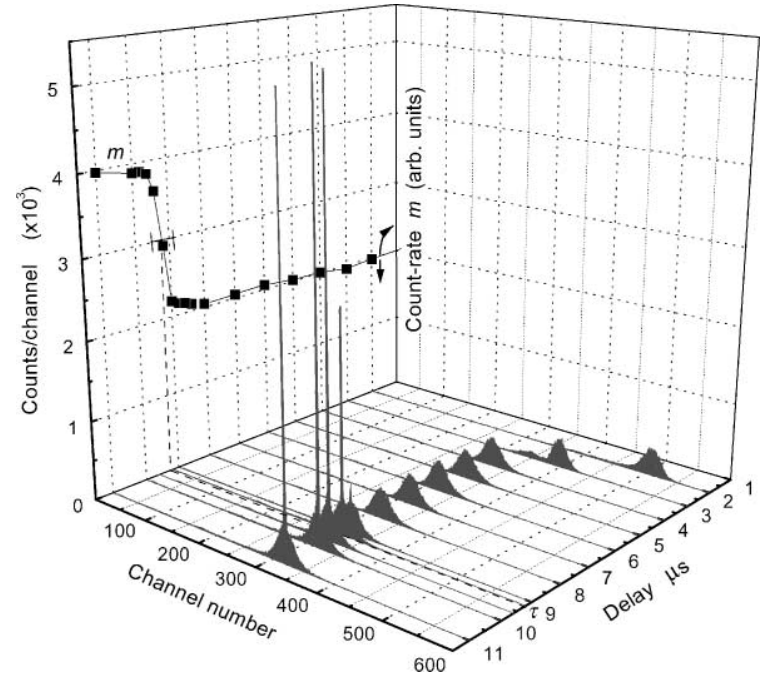

Fig. 5. Pulse-height spectra recorded by the MCA, as a function of the delay of the electronic pulses. Count-rate ( in arbitrary units. Pulse amplitude is $3.3 \mathrm{~V}$.

primarily dependent on the time resolution of the pulses, which determines the variance in the dead time of the counting system. The overall standard uncertainty $u_{\tau}$ is estimated by quadratically adding the standard uncertainties of these contributions assuming normal (gaussian) and independent distributions. For our systems, $u_{\mathrm{t}}$ was estimated to be about 0.1 or $0.2 \mu$ s for the GPSC or the PC, respectively. $u_{\mathrm{m}}$ depends primarily on the time base of the oscilloscope display. The estimated relative standard uncertainty $u_{\tau}$ in the measurements described here varied in the $5-10 \%$ range.

The next problem is to determine the actual count-rate, after having measured the dead time under the same conditions in which the correction is to be made. This issue has been treated for the two traditional dead time behaviors, the extending and non-extending ones, and analytical correction formulas for each case are well documented [4]. However, the dead time of a real system is dependent on the different dead times of each part of the system, and each of these dead times cannot be undoubtedly associated to one of the usual standard types $[5,7,14]$. Since no general solution seems to exist for the dead time problem, the behavior of the counting systems used was investigated under our conditions.
A modification of the present method was used to examine the dead time behavior of the counting system. Using a second pulse generator also triggered by the detector pulses with a delay selected independently from the first pulse generator, we were able to see if a unresolved pulse influences the system dead time by paralyzing it (extending or paralyzable behavior) or if it has no effect at all (non-extending or non-paralyzable behavior). Fixing the delay $d_{1}$ between the detector pulses and the first electronic pulses to a value shorter than the counting system effective dead time, we warrant that the first electronic pulses are not resolved from the detector pulses. Then we can inspect the counting system behavior by varying the delay $d_{2}$ between the detector and the second electronic pulses. We can thus see when the counting system becomes able to resolve a third event, if the second event increases the dead time of the system and by how long.

Fig. 6 presents the results obtained for the behavior of the PD-EC counting system, for three values of $d_{1}$ shorter than the dead time of the system. As the results show, the first electronic pulses extend the time during which the counting system cannot resolve another event. Moreover it is extended by a time interval of approximately $\tau$,

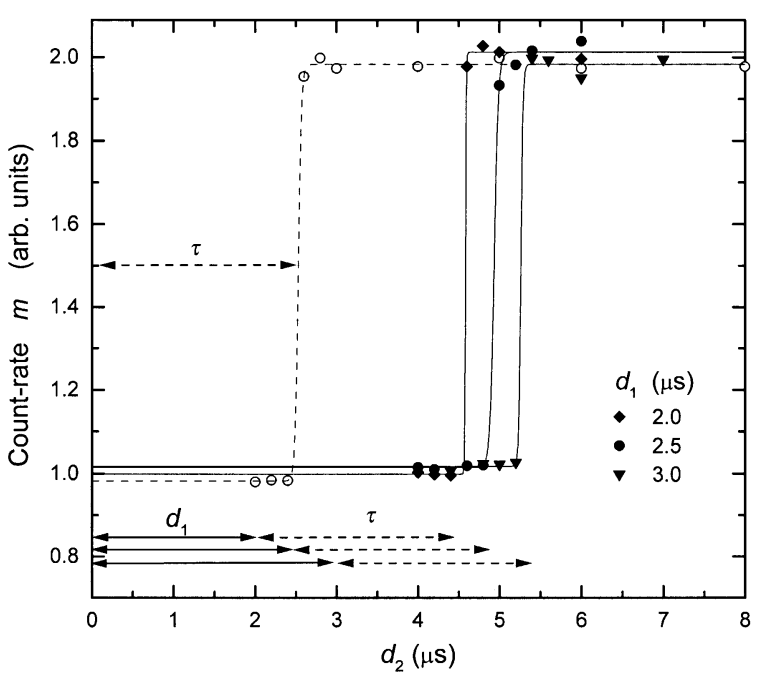

Fig. 6. Dead times $(\bigcirc)$ for a PD-EC counting system with a pulse amplitude of $2.8 \mathrm{~V}$. Behavior of this counting system, see text, with $d_{1}=2.0 \mu \mathrm{s}(\bullet), d_{1}=2.5 \mu \mathrm{s}(\bullet)$ and $d_{1}=3.0 \mu \mathrm{s}(\mathbf{\nabla})$. 
measured for the first electronic pulses. When we varied the $d_{2}$, the transition in $m$ occurred at $d_{2} \cong d_{1}+\tau$. Thus the PD-EC system exhibits clearly an extending behavior.

Using the PD-EC system dead time, measured with this method, and applying the well-known formula for the corrected count-rate of a Poisson distribution affected by a dead time of an extending behavior type [4], the actual count-rate is determined. Several tests were performed by fixing the radiation source and measuring the actual count-rate in different conditions. When we switched from the PC to the GPSC mode of the hybrid detector, the actual count-rates determined with this method agree within a standard deviation of about $0.25 \%$. Also, modifying the pile-up pulse properties by changing the shaping times of the amplifier to 0.2 and $2 \mu \mathrm{s}$, also yielded agreeing results within $0.15 \%$.

The behavior exhibited by the MCA counting system is rather more complicated. According to our results, the MCA dead time exhibits a mixed extending and non-extending behavior: during a short time interval it is extending but after that it becomes non-extending. This behavior results from the way the MCA processes the pulses. The simple cases of extending and non-extending behaviors cannot be therefore applied to this case.

\section{Conclusions}

The new DMP method for the experimental measurement of dead times is presented. This method provides a way to measure directly the counting system effective dead time due to both the dead time characteristics of the components of the pulse-processing chain as well as pulse pile-up effects, dependent on the detector pulse time properties. The method is low time consuming and hardly requires extra instrumentation, being thus convenient for the use in standard experimental situations. We achieved standard uncertainties in the dead times measured of $5-10 \%$.
When higher accuracies are required, this simple method can be no longer used. In this case, the known method based on the insertion of a dead time unit in the counting chain with a well-known behavior type (extending or non-extending) and an accurately defined dead time value (sufficiently larger than the dead time of any other unit of the counting chain) is required.

\section{Acknowledgements}

This work was carried out in the Atomic and Nuclear Instrumentation Group of the Instrumentation Centre (Unit 217/94) of the Physics Department, University of Coimbra. This work was supported by project CERN/P/FIS/15201/1999. The authors wish to thank P.C.P.S. Simões for helpful discussions.

\section{References}

[1] O.C. Johns, M.J. Yaffe, Nucl. Instr. and Meth. A 255 (1987) 559.

[2] M. Wiernik, Nucl. Instr. and Meth. 95 (1971) 13.

[3] B.A. Faddegon, Nucl. Instr. and Meth. B 51 (1990) 431.

[4] G.F. Knoll, Radiation Detection and Measurements, 2nd Edition, Wiley, New York, 1989.

[5] E. Funck, Nucl. Instr. and Meth. A 245 (1986) 519.

[6] E. Schönfeld, H. Janssen, Nucl. Instr. and Meth. A 339 (1994) 137

[7] J.W. Müller, Nucl. Instr. and Meth. 112 (1973) 47.

[8] M.G. Strauss, L.L. Sifter, F.R. Lenkszus, R. Brenner, IEEE Trans. Nucl. Sci. NS-15 (1968) 518.

[9] O.U. Anders, Nucl. Instr. and Meth. 68 (1969) 205.

[10] M. Wiernik, Nucl. Instr. and Meth. 96 (1971) 325.

[11] T. Fazzini, G. Poggi, P. Sona, N. Taccetti, Nucl. Instr. and Meth. A 356 (1995) 319.

[12] V. Drndarevic, Meas. Sci. Technol. 5 (1994) 1573.

[13] V. Drndarevic, P. Ryge, T. Gozani, Nucl. Instr. and Meth. A 277 (1989) 532.

[14] J.W. Müller, Nucl. Instr. and Meth. A 301 (1991) 543.

[15] F.L.R. Vinagre, C.A.N. Conde, Nucl. Instr. and Meth. A 450 (2000) 365. 